\title{
Comparative effects of entomopathogenic fungi and nematodes and bacterial supernatants against rice white tip nematode
}

\author{
Adnan Tülek', i I ker Kepenekçi ${ }^{2}$ Erçin Oksal ${ }^{3^{*}}$ (i) and Selçuk Hazir ${ }^{4}$
}

\begin{abstract}
The rice white tip nematode, Aphelenchoides besseyi, is the primarily important seed-borne pest of rice and was first recorded in Turkey in 1995. These biological agents, such as an entomopathogenic fungus (EPF), entomopathogenic nematodes (EPN), and bacterial supernatants, have been used against other plant parasitic nematodes but as far as not against $A$. besseyi. Accordingly, the EPF species, Purpureocillium lilacinum, and the four EPN species, Heterorhabditis bacteriophora, Steinernema carpocapsae, S. glaseri, and S. feltiae, and the supernatant of the two bacterial symbionts, Photorhabdus luminescens and Xenorhabdus bovienii, were used against A. besseyi. In addition, infected Galleria mellonella cadavers with $S$. feltiae were evaluated as a biological agent to the white tip nematode. The percentage of observed white tip symptoms, decrease in kernel numbers in the panicles, and decrease in panicle weight were evaluated. Evaluation of these parameters showed that $X$. bovienii supernatant, S. feltiae infective juveniles, and $P$. lilacinum at $10^{8}$ conidia $\mathrm{ml}^{-1}$ consistently suppressed the $A$. besseyi population.
\end{abstract}

Keywords: Aphelenchoides besseyi, Rice white tip nematode, Purpureocillium lilacinum, Entomopathogenic nematodes, Bacterial supernatants, Biological control

\section{Background}

The rice white tip nematode, Aphelenchoides besseyi, is one of the most important seed-borne pests of rice, Oryza sativa L., throughout the world. Its economic importance can vary by locations, regions, and countries, and widespread infection is possible. The yield losses fluctuate with the rate of infection, cultural practices, temperature, season of the year, cultivar, and other variable factors. In fields where all plants have been attacked, maximum losses of up to $70 \%$ for the most susceptible cultivars and of $20 \%$ for the most resistant cultivars have been reported (Prot, 1992). The yield losses have reached up to $57.9 \%$ for susceptible cultivar

\footnotetext{
* Correspondence: ercin.oksal@inonu.edu.tr

Presented at the International Conference on Biopesticides 7 (ICOB 7) and published as short summaries

${ }^{3}$ Department of Plant Protection, Faculty of Agriculture, Inonu University, 44000 Battalgazi, Malatya, Turkey

Full list of author information is available at the end of the article
}

Halilbey in fields having 77\% plants with white tips on the infected flag leaves (Tulek and Cobanoglu, 2010).

The most effective and acceptable management for $A$. besseyi is hot water treatment of the rice seeds (EPPO, 2012). However, this approach requires (1) investing in expensive equipment and expertise and (2) maintaining the water temperature at a level that adversely affects the nematodes without affecting the viability of the seeds. Thus, other management approaches need to be explored such as development of resistant cultivars and chemical seed treatments. Unfortunately, commercial resistant cultivars have not yet been developed and studies have showed that chemical treatments can negatively affect seed germination and do not result in nematode mortality (Hui et al., 2014). For these reasons, control of $A$. besseyi is difficult mainly due to the ineffectiveness of the conventional pesticides.

Alternatives to pesticides such as entomopathogenics for the control of plant parasitic nematodes are receiving considerable attention because they are environmentally 
more acceptable than chemicals (Arıcı et al., 2012). In previous studies, different biological control methods were evaluated to determine the suppressive effects of entomopathogenic fungi (EPFs), especially Purpureocillium lilacinum TR1 (syn: Paecilomyces lilacinus), and the supernatants of the in vitro cultures of mutualistic bacteria and their entomopathogenic nematodes (EPNs) against plant parasitic nematodes such as the root-knot (RKN), sting, and ring nematodes. Some studies reported successful reductions in RKN populations and positive effects on plant development (Grewal et al., 1999; Kepenekci et al., 2015).

Purpureocillium is monotypic, containing the single species $P$. lilacinum. It is a common filamentous fungus and saprotroph. P. lilacinum has been isolated from a wide range of habitats including insects, forestsgrassland, cultivated and uncultivated soils sediments, deserts, and sewage sludge and from females of root-knot and cyst nematodes and their eggs (Fiedler and Sosnowska, 2007). It has shown promising results as a biocontrol agent against the destructive RKNs (Stirling and West, 1991). Laboratory, glasshouse, and field trials of P. lilacinum have shown that some isolates are aggressive parasites while others, though morphologically indistinguishable, are less or non-pathogenic to nematodes and insects. In some cases, promising isolates have failed to provide a control in the field, while they were effective in glasshouse trials (Gomes-Carniero and Cayrol, 1991).

EPNs in the genus Heterorhabditis (Heterorhabditidae) and Steinernema (Steinernematidae) are obligate organisms that infect a wide range of insect hosts (Kaya and Gaugler, 1993). The infective juvenile (IJ) stage of EPNs is associated mutualistically with bacteria in the genus Photorhabdus spp. (heterorhabditids) or Xenorhabdus spp. (steinernematids) (Boemare, 2002). Symbiotic bacteria produce several toxins, antibiotics, and other chemicals that can be toxic to many species of nematodes, including those in the genera Meloidogyne and Caenorhabditis, and mainly prevent other microorganisms from colonizing the host cadaver (Boemare, 2002). In previous studies, efficacy of different EPN species and their bacterial metabolites, tested primarily on RKNs, showed a number of successful results (Lewis and Grewal, 2005).

The main objectives of this study were to determine the efficacy of the EPF, P. lilacinum, the four EPN species, and the supernatants of their mutualistic bacteria on A. besseyi.

\section{Materials and methods}

\section{Plant parasitic nematode}

Aphelenchoides besseyi was isolated from the infested seed of cultivar Halilbey and reared on a carrot culture as described by Tulek et al. (2009). Briefly, newly harvested carrots were peeled in a laminar flow hood and then surface sterilized in ethanol (95\%) for $5 \mathrm{~min}$. The alcohol was evaporated by a gentle flame with the Bunsen burner, and the carrots were peeled again and cut into $1-\mathrm{cm}$-thick segments. Three segments were placed in a 9-cm-diameter Petri dish. A. besseyi individuals were collected from rice panicles by using a modified Baermann funnel method, and the surfaces of the nematodes were sterilized with streptomycin sulfate $(0.1 \%)$ for $10 \mathrm{~min}$. One surfacesterilized female was transferred onto a carrot segment in each Petri dish. The plates were covered tightly with parafilm and placed in an incubator at $22 \pm 1{ }^{\circ} \mathrm{C}$ to allow the nematodes to develop and reproduce. Four months later, a mixture of nematode stages was harvested by adding a sufficient amount of sterile water to the Petri dishes. The nematodes were collected from the water, then concentrated by passing through a $20-\mu \mathrm{m}$ sieve. The collected $A$. besseyi were stored in the refrigerator at $5{ }^{\circ} \mathrm{C}$ until have been used in experiments.

\section{Entomopathogenic fungi}

Purpureocillium lilacinum isolated from RKNs in tomato roots was obtained from the culture collection in Ankara Plant Protection Central Research Institute. A Turkish isolate of $P$. lilacinum was identified by classical (morphologic and morphometric properties) and molecular methods (Kepenekci et al., 2015). The fungus was subcultured on a potato dextrose agar and incubated at 25 $\pm 1{ }^{\circ} \mathrm{C}$ for 14 days. The conidia were collected using a sterilized rubber loop attached to 1-ml borosilicate pipette. The scraped material was suspended in sterile water containing $0.05 \%$ Tween- 80 and stored at $4{ }^{\circ} \mathrm{C}$. The concentration was adjusted to $1 \times 10^{6}, 1 \times 10^{7}$, and $1 \times$ $10^{8}$ conidia $/ \mathrm{ml}^{-1}$ based on hemocytometer count.

\section{Entomopathogenic nematodes}

A non-native EPN Steinernema glaseri (Belgium isolate) and native EPNs, black sea isolate of $S$. carpocapsae in the Rize province, Heterorhabditis bacteriophora (isolate 09-43) in the Aydin province, and S. feltiae (isolate 09-31) in the Aydin province were used in the experiments. Last instar larvae of wax moth (Galleria mellonella, Lepidoptera: Pyralidae) were used to culture nematodes at room temperature $\left(23-24{ }^{\circ} \mathrm{C}\right)$ as described by Kaya and Stock (1997).

The IJs emerging from cadavers were harvested from nematode-infected larvae, which were placed on white traps (White, 1927). The IJs were collected and rinsed in sterile distilled water for three times. Using a Tetra Pak juice box, each species was kept separately before refrigerating at $10^{\circ}$ C. The harvested IJs were used within 2 weeks after emergence for the experiments (Gülcü and Hazır, 2012).

\section{Preparation of insect cadavers containing IJs}

Besides, using free living IJs, insect cadavers containing S. feltiae (referred to as infected cadavers) were tested as 
well. Five G. mellonella larvae were placed into $9-\mathrm{cm}$ plastic Petri dishes lined with a filter paper and exposed to 500 IJs for 2 days. Cadavers with the nematode/bacterium complex were transferred to a moist environment for 3 days before they were used in experiments.

\section{Preparation of bacterial supernatant}

To isolate the symbiotic bacteria of EPNs, approximately 500 IJs of $H$. bacteriophora or S. feltiae were surfacesterilized with $0.4 \%$ Hyamine solution for 3-4 min and rinsed with sterile distilled water for three times. The IJs were crushed in a 2-ml sterile Eppendorf tubes using pestles (Somvanshi et al., 2006). A drop of suspension was streaked onto a nutrient agar [containing 0.025\% $(w / v)$ bromothymol blue medium and $0.004 \%(w / v)$ triphenyl-tetrazolium chloride] with a sterile loop and incubated at $28^{\circ} \mathrm{C}$. A single bacterial colony of $X$. bovienii or P. luminescens kayaii was selected and transferred into Soybean Casein Digest Medium (TSB, Tryptic Soy Broth) and placed in a shaking incubator set at $180 \mathrm{rpm}$ for $192 \mathrm{~h}$ at $28{ }^{\circ} \mathrm{C}$. To obtain the cell-free supernatant, bacterial cultures were centrifuged at $20,000 \mathrm{~g}$ at $4{ }^{\circ} \mathrm{C}$ for 15 min (SIGMA model K-30) (Gulcu et al., 2012).

\section{Pot experiments in the field}

These experiments were conducted in plastic pots $(40 \times$ $60 \mathrm{~cm}$ ) at Thrace Agricultural Research Institute in Edirne, Turkey. The pots were kept in the field during the 2013 rice-growing season. Osmanck rice cultivar (moderately susceptible to the white tip nematode) was used in all experiments.

Each pot contained $5000 \mathrm{~cm}^{3}$ steam-sterilized soil (mixtures of $95 \%$ clay loam, $5 \%$ organic matter), and 20 rice seeds were sowed. The plants were arranged as 15 plants per pot after plant emergence. When the plants were about $3 \mathrm{~cm}$ in height, $500 \mathrm{IJs}$ of $A$. besseyi (mixture of juveniles and adults) in $50 \mathrm{ml}$ distilled water were inoculated into each pot. There were 11 treatments with each pot in each treatment receiving the following rate of conidia, EPN, insect cadavers with S. feltiae, or bacterial supernatant:

For each EPF treatment, a conidial concentration of $P$. lilacinum in $50 \mathrm{ml}$ distilled water was applied to the pots as follows:

1. $10^{6}$ conidia $\mathrm{ml}^{-1}$

2. $10^{7}$ conidia $\mathrm{ml}^{-1}$

3. $10^{8}$ conidia $\mathrm{ml}^{-1}$

For each EPN species, the rate of $25 \mathrm{IJs} \mathrm{cm}^{-2}$ (pot)

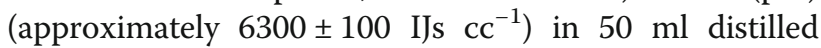
water was applied as follows:

4. S. feltiae

5. S. glaseri
6. S. carpocapsae

7. H. bacteriophora

8. Four 5-day-old insect cadavers containing S. feltiae were buried $1 \mathrm{~cm}$ beneath the soil surface in each pot.

9. Ten milliliters of bacterial supernatant of $X$. bovienii was injected into the soil with a syringe.

10.Ten milliliters of bacterial supernatant of $P$. luminescens was injected into the soil with a syringe.

11.Control pots had only 500 IJs of $A$. besseyi.

During and after the inoculations, the water depth in each pot was maintained at $2-3 \mathrm{~cm}$. Then, according to the plant growth, the water depth was maintained at 4$5 \mathrm{~cm}$. All treatments were arranged in a randomized complete block design with five replicates. The plants were harvested 120 days post-treatment. The percentage of white tip symptoms, decrease in kernel numbers in the panicles, and decrease in panicle weight were recorded. During the flowering stage, the ratios of plants (\%) with symptoms of white tip in the flag leaf were determined in each pot. On the tillers of the affected plants, the tips of the leaves whiten for a distance of 3 to $5 \mathrm{~cm}$ and then die off. To calculate the percentage decrease of panicle weight and kernel number, 10 plants with and without white tip symptoms in each pot were harvested and their panicle weight and kernel numbers were determined individually. Then, the proportion of their panicle weights and kernel numbers were used.

\section{Statistical analysis}

Statistical analysis was carried out by "JMP 5.0.1" software. Treatment effects were analyzed using one-factor ANOVA process, and means were separated by Tukey's test that is sized for all differences among the means at $P<0.05$ level (Tukey, 1953).

\section{Results and discussion}

For the percentage of white tip symptoms, $X$. bovienii supernatant and $P$. lilacinum at $10^{8}$ conidia $\mathrm{ml}^{-1}$ treatments were not significantly different than P. lilacinum at $10^{6}$ conidia $\mathrm{ml}^{-1}$ treatment which had the lowest percentage of white tip symptoms (Fig. 1). However, in two out of three parameters (percent decrease in kernel numbers and percent decrease in panicle weight) measured, the $X$. bovienii supernatant and P. lilacinum at $10^{8}$ conidia $\mathrm{ml}^{-1}$ treatments had the greatest effect on reducing $A$. besseyi infestation, whereas the other treatments gave more variable results (Figs. 2 and 3).

Figure 1 shows that the lowest percentage of plants with white tip symptoms was obtained from the P. lilacinum $10^{6}$ treatment (19\%), whereas the highest percentage symptoms was observed in the $P$. luminescens treatment (60\%), followed by S. carpocapsae (57\%) and control (51\%), respectively. Interestingly, among P. lilacinum (all 


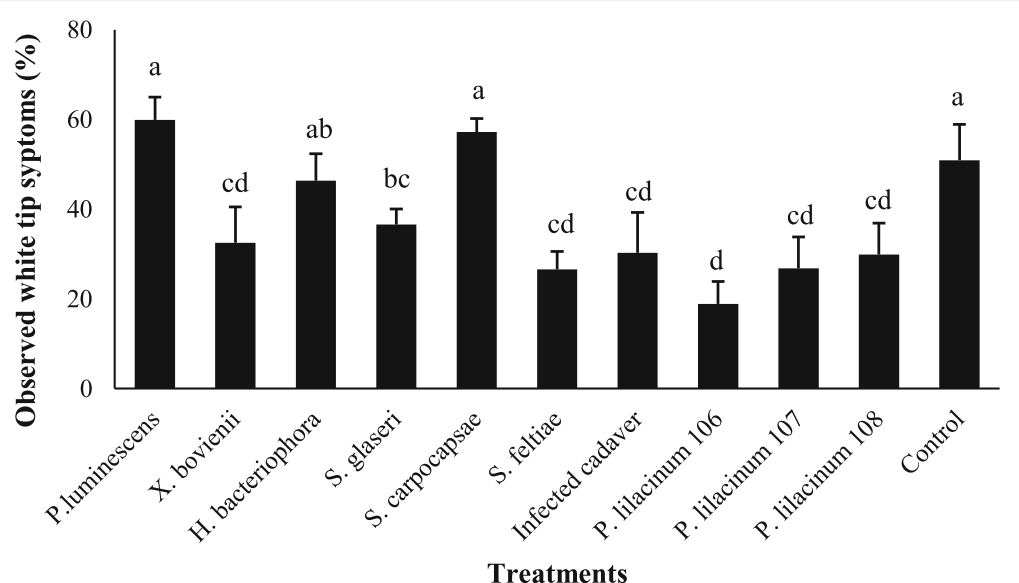

Fig. 1 The effect of treatments on the percentage of white tip symptoms observed on the rice plants. Differences were significant at $P<0.05$, and the same letters are not significantly different

treatments), infected cadaver, S. feltiae, and X. bovienii treatments showed no significant differences, but all these treatments exhibited statistically less white tip symptoms than $P$. luminescens, S. carpocapsae, control, and $H$. bacteriophora $(F=7.33$; $d f=10,54 ; P<0.05)$ (Fig. 1$)$.

The decrease of total panicle weight was the lowest at the $X$. bovienii treatment (8\%) and the highest at the $P$. lilacinum $10^{6}$ treatment (44\%) (Fig. 2). Except at the $P$. lilacinum $10^{8}$ and S. feltiae treatments, there was a significant difference between $X$. bovienii and the other treatments including control $(F=3.69 ; d f=10,54 ; P<0.05)$ (Fig. 2). The P. lilacinum $10^{8}$ and S. feltiae treatments were not significantly different from each other.

For the percent decrease in kernel numbers, the $X$. bovienii supernatant treatment exhibited the strongest effect resulting in an average of $9 \%$ loss in kernels in each panicle, followed by $10 \%$ in the infected cadaver, $10 \%$ for S. feltiae IJs, and $22 \%$ for P. lilacinum $10^{8}$ in treatments
(Fig. 3). No statistically differences were observed among these treatments, but there was a significant difference between $X$. bovienii and all other treatments and control $(F$ $=2.97 ; d f=10,54 ; P<0.05)$ (Fig. 3). However, the infected cadaver, S. feltiae IJs, and P. lilacinum $10^{8}$ treatments were not significantly different than the other treatments, and the P. lilacinum $10^{8}$ treatment was not significantly different than the other 10 treatments (Fig. 3).

Our findings indicated that several treatments had a significant negative effect on the rice white tip nematode. Evaluation of the treatments on three parameters showed that $X$. bovienii supernatant, $S$. feltiae IJs, and P. lilacinum at $10^{8}$ conidia $\mathrm{ml}^{-1}$ consistently had the least effect on percentage decrease of kernel numbers and panicle weight loss as well as on white tip symptoms. The other treatments gave variable results although some of the treatments resulted in significant negative effects on the nematode for one or two of the parameters. For example,

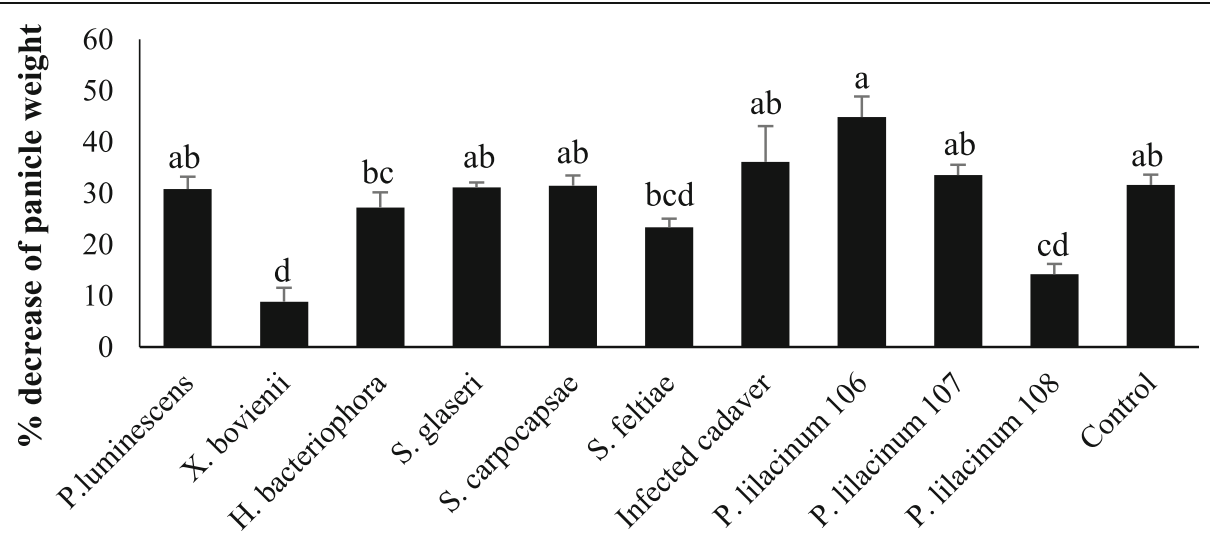

Treatments

Fig. 2 The effect of treatments on the average decrease of panicle weight (\%). Differences were significant at $P<0.05$, and the same letters are not significantly different 


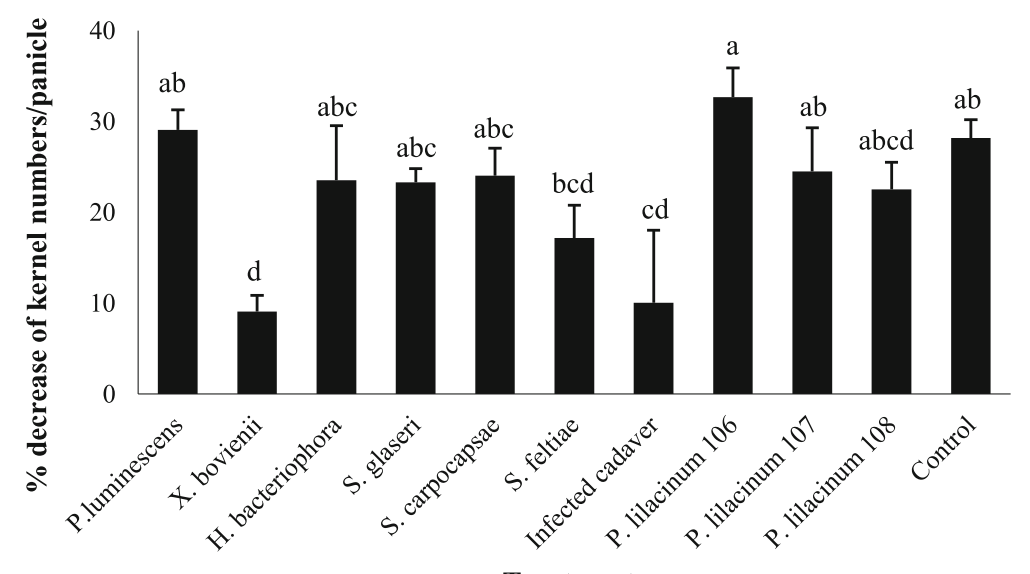

Treatments

Fig. 3 The effect of treatments on the average decrease of kernel numbers (\%) in the panicles. Differences were significant at $P<0.05$, and the same letters are not significantly different

the infected cadaver treatment was equally effective as $X$. bovienii supernatant, S. feltiae IJs, and P. lilacinum at $10^{8}$ conidia $\mathrm{ml}^{-1}$ on the nematode for percentage decrease of kernel numbers and white tip symptoms but not for percentage decrease on panicle weight.

Previously, different EPN species and their bacterial suspensions with different application methods have been tested on RKNs (M. incognita, M. arenaria, $M$. javanica, etc.), the ring nematodes, Criconemella spp., and the sting nematode, Belonolaimus longicaudatus. Most of these studies showed positive results, but some of them exhibited no beneficial effects (Grewal et al., 1999). Recently, Kepenekci et al. (2015) evaluated the suppressive effects of IJs and infected cadavers of four EPN species (S. glaseri, S. carpocapsae, S. feltiae, and $H$. bacteriophora) and two application methods (injection to soil and plant dipping) of $X$. bovienii or P. luminescens supernatants on the RKNs, $M$. incognita and $M$. arenaria, on tomatoes. Although the infected cadaver applications of EPNs and the aqueous suspensions of IJs suppressed the negative effects of RKNs, the most effective results were obtained from the dipping method of tomato roots in the $X$. bovienii supernatant. Also, our findings were similar in that the $X$. bovienii bacterial supernatant was one of the most consistent treatments, which resulted in the suppression of the negative effects of $A$. besseyi on rice plants. One common thread among the EPN species tested for control of plant parasitic nematodes has shown that $S$. feltiae was the most reliable in providing some level of control (Lewis and Grewal, 2005). Several mechanisms of nematicidal activity have been offered to explain how EPNs could have an effect on plant parasitic nematodes. Various predator/prey interactions may affect nematode populations indiscriminately (Ishibashi and Kondo, 1986). Both EPNs and plant parasitic nematodes are attracted to $\mathrm{CO}$, produced by plant roots. EPNs may physically interfere with root invasion and feeding activities of plant parasitics (Bird and Bird, 1986). Grewal et al. (1999) showed that the $S$. feltiae associated with $X$. bovienii had a key role for the suppression of RKNs.

The highest rate (108 conidial concentration) of this fungus was as effective as that of $X$. bovienii supernatant for having minimal effects on percentage kernel loss and panicle weight. However, all rates of P. lilacinum were effective in reducing white tip symptoms. We hypothesize that there was no correlation between white tip symptoms and kernel loss or panicle weight.

Although there are no reports on the effect of P. lilacinum on $A$. besseyi, this fungus was applied to soil to control nematodes that attack plant roots (Esser and ElGholl, 1993). In other studies involving P. lilacinum and plant parasitic nematodes, Khalil et al. (2012) evaluated the efficacy of azadirachtin $0.15 \%$, azadirachtin $0.03 \%$, oxamyl, Pseudomonas fluorescens, Bacillus subtilis, P. lilacinum, and abamectin against the RKN, M. incognita, on potted tomato plants. They showed that P. lilacinum (bio-nematon) was the most effective treatment for both egg and gall masses achieving 76.9 and $88.2 \%$ reduction, respectively. Siddiqui et al. (2000) demonstrated that $P$. lilacinum parasitized both eggs and females of $M$. javanica and that ethyl acetate extract of $P$. lilacinum and $P$. aeruginosa caused 100 and $64 \%$ mortality of $M$. javanica larvae, respectively, after $24 \mathrm{~h}$.

\section{Conclusions}

For future studies, control of plant parasitic nematodes and the combined application of $P$. lilacinum and $X$. bovienii supernatant could potentially be feasible and much more effective on $A$. besseyi. 


\section{Acknowledgements}

We are grateful to Dr. Harry K. Kaya (University of California, Davis Department of Entomology and Nematology) for editing the manuscript.

\section{Authors' contributions}

All authors read and approved the final manuscript.

\section{Competing interests}

The authors declare that they have no competing interests.

\section{Publisher's Note}

Springer Nature remains neutral with regard to jurisdictional claims in published maps and institutional affiliations.

\section{Author details}

${ }^{1}$ Thrace Agricultural Research Institute, 22100 Edirne, Turkey. ${ }^{2}$ Department of Plant Protection, Faculty of Agriculture, Gaziosmanpaşa University, 60250 Tokat, Turkey. ${ }^{3}$ Department of Plant Protection, Faculty of Agriculture, Inonu University, 44000 Battalgazi, Malatya, Turkey. ${ }^{4}$ Department of Biology, Faculty of Arts and Sciences, Adnan Menderes University, 09100 Aydın, Turkey.

Received: 26 July 2017 Accepted: 6 December 2017

Published online: 30 January 2018

\section{References}

Arıcı SE, Gülmez I, Demirekin H, Zahmekıran H, Karaca I (2012) Efficiency of entomopathogenic fungus Fusarium subglutinans against Aphis fabae Scopoli (Hemiptera: Aphididae). Turk Biyo Muc Derg 3:89-96

Bird AF, Bird J (1986) Observations on the use of insect parasitic nematodes as means of biological control of root-knot nematodes. Int J Parasitol 16(5):511-516

Boemare, N.E. 2002. Biology, taxonomy and systematics of Photorhabdus and Xenorhabdus. Pp. 35-56 in R. Gaugler, ed. Entomopathogenic Nematology. Wallingford, UK: CABI Publishing

EPPO, 2012. Aphelenchoides besseyi, Prepared by CABI and EPPO for the EU under Contract 90/399003

Esser, R.P. and El Gholl, N.E. 1993. Paecilomyces lilacinus, a fungus that parasitizes nematode eggs. Floride Department of Agriculture, Division of Plant Industry, Nematology Circular 203

Fiedler Z, Sosnowska D (2007) Nematophagous fungus Paecilomyces lilacinus (Thom.) Samson is also a biological agent for control of greenhouse insects and mite pests. BioControl 52:547-558

Gomes Carniero RMD, Cayrol J (1991) Relationship between inoculum density of the nematophagous fungus Paecilomyces lilacinus and control of Meloidogyne arenaria on tomato. Rev Nématol 14(4):629-634

Grewal PS, Lewis EE, Venkatachari S (1999) Allelopathy: a possible mechanism of suppression of plant-parasitic nematodes by entomopathogenic nematodes. Nematology 1:735-743

Gülcü, B. and Hazır S. 2012. An alternative storage method for entomopathogenic nematodes. Turk J Zool., 2012; 36 (4): 562-565

Gulcu B, Hazir S, Kaya HK (2012) Scavenger deterrent factor (SDF) from symbiotic bacteria of entomopathogenic nematodes. J Invertebr Pathol 110(2012):326-333

Hui F, Li-Hui W, Mao-Song L, Yi-jun Z (2014) Assessment of rice cultivars in China for field resistance to Aphelenchoides besseyi. J Integr Agric 13(10):2221-2228

Ishibashi N, Kondo E (1986) Steinernema feltiae (DD-136) and S. glaseri: persistence in soil and bark compost and their influence on native nematodes. J Nematol 18(3):310-316

Kaya HK, Gaugler R (1993) Entomopathogenic nematodes. Annu Rev Entomol 38:181-206

Kaya, H.K. and Stock, S.P. 1997. Techniques in insect nematology. Manual of techniques in insect pathology. In: Lacey L, editor. San Diego, CA: Academic Press; 1997. pp. 281-324

Kepenekci I, Hazir S, Lewis EE (2015) Evaluation of entomopathogenic nematodes and the supernatants of the in vitro culture medium of their mutualistic bacteria for the control of the root-knot nematodes Meloidogyne incognita and M. arenaria. Pest Manag Sci 72(2):327-334

Kepenekci I, Oksal E, Sağlam HD, Atay T, Tülek A, Evlice E (2015) Identification of Turkish isolate of entomopathogenic fungi, Purpureocillium lilacinum (syn: Paecilomyces lilacinus) and its effect on potato pests, Phthorimaea operculella (Zeller) (Lepidoptera: Gelechiidae) and Leptinotarsa decemlineata (Say) (Coleoptera: Chrysomalidae). Egypt J Biol Pest Control 25(1):121-127
Khalil MSH, Allam AFG, Barakat AST (2012) Nematicidal activity of some biopesticide agents and microorganisms against root-knot nematode on tomato plants under greenhouse conditions. J Plant Prot Res 52(1):47-52

Lewis EE, Grewal PS (2005) Interactions with plant parasitic nematodes. In: By Grewal PS, Ehlers RU, Shapiro-llan DI (eds) Nematodes as biocontrol agents. CABI Publishing, New York, pp 349-362

Prot, J.C. 1992. White tip. In: Webster RK, Gunnel PS (eds.). Compendium of rice diseases. A.P.S. Press, University of California, Davis, CA, USA, pp. 46-47

Siddiqui IA, Qureshi SA, Sultana V, Ehteshamul-Haque S, Ghaffar A (2000) Biological control of rot-root knot disease complex of tomato. Plant Soil 227:163-169

Somvanshi VS, Lang E, Ganguly S, Swiderski J, Saxena AK, Stackebrandt E (2006) A novel species of Xenorhabdus, family Enterobacteriaceae: Xenorhabdus indica sp. nov., symbiotically associated with entomopathogenic nematode Steinernema thermophilum Ganguly and Singh, 2000. Syst Appl Microbiol 29:519-525

Stirling GR, West LM (1991) Fungal parasites of root-knot nematode eggs from tropical and sub-tropical regions of Australia. Australas Plant Pathol 20(4):149-154

Tukey, J. 1953. A problem of multiple comparisons. Dittoed manuscript of 396 pages, Princeton University

Tulek A, Cobanoglu S (2010) Distribution of the rice white tip nematode, Aphelenchoides besseyi, in rice growing areas in Thrace region of Turkey. Nematol Mediterr 38:215-217

Tulek, A., Kepenekci, I., Cobanoglu, S., Hekimhan, H., Devran, Z., Melik, B. and Elekcioglu, H.I. 2009. A new culturing method for the rice white tip nematode, Aphelenchoides besseyi Christie, 1942, on carrot discs. Russ J Nematol., 2009, 17 (2), 135 - 136

White GF (1927) A method for obtaining infective nematode from cultures. Sciences 66:302-303

\section{Submit your manuscript to a SpringerOpen ${ }^{\circ}$ journal and benefit from:}

- Convenient online submission

- Rigorous peer review

- Open access: articles freely available online

- High visibility within the field

- Retaining the copyright to your article

Submit your next manuscript at $>$ springeropen.com 